\title{
Asymmetry models based on ordered score and separations of symmetry model for square contingency tables
}

\author{
Shuji Ando \\ Department of Information and Computer Technology, Faculty of Engineering, \\ Tokyo University of Science, Katsushika-ku, Tokyo, 125-8585, Japan, \\ e-mail: shuji.ando@rs.tus.ac.jp
}

\section{SUMMARY}

\begin{abstract}
This study proposes two original asymmetry models based on ordered scores for square contingency tables with the same row and column ordinal classifications. The proposed models can be applied to cases in which the scores of all categories are known or unknown. In the proposed models, the log odds for an observation falling in the $(i, j)$ th cell instead of the $(j, i)$ th cell are inversely proportional to the difference of the ordered scores corresponding to categories $i$ and $j$. The asymmetry parameter of the proposed model can be useful for inferring whether the row variable is stochastically greater than the column variable or vice versa. The proposed models constantly hold when the symmetry model holds, but the converse is not necessarily true. This study also examines what is necessary for a model, in addition to the proposed models, to satisfy the symmetry model, and gives separations of the symmetry model using the proposed and marginal mean equality models. We apply real data to show the utility of the proposed models. The proposed models provide a better fit than that of the existing models.
\end{abstract}

Key words: asymmetry, midpoint, necessary and sufficient condition, ordered category, power parameter, ridit, symmetry

\section{Introduction}

This study proposes two original asymmetry models based on known and unknown ordered scores for square contingency tables with the same row and column ordinal classifications. Specifically, this study focuses on two types of data in which (i) the scores of all categories are known, and (ii) the scores of all categories are unknown. A typical example of type (i) is a categorical variable set as an interval based on a continuous variable. In this 
case, Graubard and Korn (1987) and Senn (2007) recommended assigning ordered scores as midpoints of category intervals (midpoint scores), instead of equally spaced scores.

Table 1 is taken directly from Tomizawa et al. (2006). This dataset examined the number of decayed teeth of 349 male 18-39-year-old patients of a dental clinic in Sapporo City, from 2001 to 2005. The number of decayed teeth on the right and left sides were categorized as " 0 to 4 ", "5 to 8", and "9 and above". The ordered scores are assigned as the midpoint scores (0-4: 2; 5-8: 6.5; 9 and above: 12), assuming that the maximum numbers of teeth on the left and right sides are 15 .

Table 1. Decayed teeth of 349 male 18-39-year-old patients of a dental clinic in Sapporo City, for the period 2001-2005; from Tomizawa et al. (2006). (The parenthesized values are the maximum likelihood estimates of the expected frequencies under the Another Ordinal Quasi-Symmetry model.)

\begin{tabular}{|c|c|c|c|c|}
\hline \multirow[b]{2}{*}{$\begin{array}{c}\text { Left } \\
\text { (number of decayed teeth) }\end{array}$} & \multicolumn{3}{|c|}{ Right (number of decayed teeth) } & \multirow[b]{2}{*}{ Total } \\
\hline & $\begin{array}{l}0 \text { to } 4 \\
(1)\end{array}$ & $\begin{array}{c}5 \text { to } 8 \\
(2)\end{array}$ & $\begin{array}{c}9 \text { and above } \\
(3)\end{array}$ & \\
\hline 0 to $4(1)$ & $\begin{array}{c}118 \\
(118.00)\end{array}$ & $\begin{array}{c}37 \\
(38.11)\end{array}$ & $\begin{array}{c}2 \\
(2.17)\end{array}$ & 157 \\
\hline 5 to $8(2)$ & $\begin{array}{c}21 \\
(19.89)\end{array}$ & $\begin{array}{c}87 \\
(87.00)\end{array}$ & $\begin{array}{c}23 \\
(21.67)\end{array}$ & 131 \\
\hline 9 and above (3) & $\begin{array}{c}2 \\
(1.83)\end{array}$ & $\begin{array}{c}11 \\
(12.33)\end{array}$ & $\begin{array}{c}48 \\
(48.00)\end{array}$ & 61 \\
\hline Total & 141 & 135 & 73 & 349 \\
\hline
\end{tabular}

A typical example of type (ii) is a case where ordered categories are not set as intervals. In this case, it is difficult to assign ordered known scores to categories.

Table 2 is taken from Tomizawa (1984). This dataset examined 4746 students of the Tokyo University of Science for unaided distance vision, in April 1982; the dataset comprised about 10\% women. The unaided distance vision of the right and left eyes is categorized as "Best", "Second", "Third", and "Worst". For this dataset, we treat the ordered scores as unknown because of the unclear cut-points of vision grades.

In the linear diagonals-parameter symmetry model proposed by Agresti (1983), the log odds for an observation falling in the $(i, j)$ th cell instead of the $(j, i)$ th cell are proportional to the difference of equally spaced scores corresponding to categories $i$ and $j$. Agresti (2002, p. 429) and Iki et al. (2009) extended this model such that it could be applied to types (i) and (ii) respectively. 
Table 2. Unaided vision data of 4746 students of the Tokyo University of Science; from Tomizawa (1984). (The parenthesized values are the maximum likelihood estimates of the expected frequencies under the Another Ridit Score Type Quasi-Symmetry model)

\begin{tabular}{rccccc}
\hline & \multicolumn{5}{c}{ Left eye grade } \\
\cline { 2 - 5 } Right eye grade & Best (1) & Second (2) & Third (3) & Worst (4) & Total \\
\hline Best (1) & 1291 & 130 & 40 & 22 & 1483 \\
& $(1291.24)$ & $(124.48)$ & $(47.89)$ & $(20.41)$ & \\
Second (2) & 149 & 221 & 114 & 23 & 507 \\
& $(154.59)$ & $(221.07)$ & $(105.18)$ & $(22.43)$ & \\
Third (3) & 64 & 124 & 660 & 185 & 1033 \\
& $(56.12)$ & $(132.86)$ & $(659.99)$ & $(196.50)$ & \\
Worst (4) & 20 & 25 & 249 & 1429 & 1723 \\
& $(21.59)$ & $(25.57)$ & $(237.44)$ & $(1428.65)$ & \\
\hline Total & 1524 & 500 & 1063 & 1659 & 4746 \\
\hline
\end{tabular}

Tomizawa (1990) proposed another linear diagonals-parameter symmetry model with a structure in which the log odds are inversely proportional to the difference of equally spaced scores. Given the context, this study aims to extend this model's applicability to datasets of types (i) and (ii).

Table 3 shows the observational values of the $\log$ odds for Tables 1 and 2 . Table 3a indicates that Table 1 may have a structure in which the log odds are inversely proportional to the difference in ordered scores. From Table $3 \mathrm{~b}$, it is unclear whether Table 2 has a structure in which the log odds are proportional (or inversely proportional) to the difference in ordered scores.

Table 3. Observational values of the log odds for Tables 1 and 2 (a) For Table 1

\begin{tabular}{rrrr}
\hline & \multicolumn{3}{c}{ Right (number of decayed teeth) } \\
\cline { 2 - 4 } Left & 0 to 4 & 5 to 8 & 9 and above \\
(number of decayed teeth) & $(1)$ & $(2)$ & $(3)$ \\
\hline 0 to 4 (1) & - & 0.57 & 0 \\
5 to 8 (2) & - & - & 0.74 \\
9 and above (3) & - & - & - \\
\hline
\end{tabular}

(b) For Table 2

\begin{tabular}{rrrrr}
\hline & \multicolumn{4}{c}{ Left eye grade } \\
\cline { 2 - 5 } Right eye grade & Best (1) & Second (2) & Third (3) & Worst (4) \\
\hline Best (1) & - & -0.14 & -0.47 & 0.10 \\
Second (2) & - & - & -0.08 & -0.08 \\
Third (3) & - & - & - & -0.30 \\
Worst (4) & - & - & - & - \\
\hline
\end{tabular}


The proposed models constantly hold when the symmetry model holds, but the converse is not necessarily true. This study also examines what is necessary for a model, in addition to the proposed models, to satisfy the symmetry model, and gives separations of the symmetry model using the proposed and marginal mean equality models.

The remainder of this paper is organized as follows. Section 2 introduces existing models based on ordered scores. Section 3 proposes two original models based on ordered scores and gives separations of the symmetry model using the proposed models. Section 4 performs the goodness-of-fit test for the proposed model. Section 5 demonstrates the utility of the proposed models by applying them to real datasets (Tables 1 and 2). Section 6 closes with concluding remarks.

\section{Reviews of models based on ordered scores and separations of symmetry}

Consider an $R \times R$ square contingency table with the same row and column ordinal classifications. We denote by $p_{i j}$ the probability that an observation will fall in the $(i, j)$ th cell of the table $(i=1, \ldots, R ; j=1, \ldots, R)$. Let $X$ and $Y$ denote the row and column variables, the marginal probabilities $p_{i}$. $\sum_{t=1}^{R} p_{i t}$ and $p_{\cdot i}=\sum_{s=1}^{R} p_{s i}$ for $i=1, \ldots, R$, and the marginal distribution functions $F_{i}^{X}=\sum_{s=1}^{i} p_{s}$. and $F_{i}^{Y}=\sum_{t=1}^{i} p_{\text {. }}$, for $i=1, \ldots, R-1$. Then, the marginal ridits are defined by

$$
r_{i}^{X}=\sum_{s=1}^{i-1} p_{s \cdot}+\frac{p_{i} .}{2} \quad \text { and } \quad r_{i}^{Y}=\sum_{t=1}^{i-1} p_{\cdot t}+\frac{p_{\cdot i}}{2} \quad(i=1, \ldots, R),
$$

see Bross (1958).

Consider a model defined as

$$
\log \left(\frac{p_{i j}}{p_{j i}}\right)=\left(u_{j}-u_{i}\right) \log \delta \quad(i<j),
$$

where $u_{k}$ is the ordered score of category $k(k=1, \ldots, R)$. It must be noted that $u_{1}<u_{2}<\cdots<u_{R}$. This model has a structure in which the log odds $\log \left(p_{i j} / p_{j i}\right)$ are proportional to $u_{j}-u_{i}$. Model (2.1) with $\delta=1$ is identical to the symmetry (S) model (see Bowker, 1948; Bishop et al., 1975, p. 282).

When $u_{k+1}-u_{k}$, for all $k=1, \ldots, R-1$, are the constant $d$ (namely, $u_{k}$ is an equally spaced score), the model (2.1) is equivalent to the linear 
diagonals-parameter symmetry (LDPS) model proposed by Agresti (1983). When $u_{k}$ can be assigned a known ordered score, such as the midpoint score, model (2.1) is equivalent to the ordinal quasi-symmetry (OQS) model proposed by Agresti (2002, p. 429). When $u_{k}$ is assigned the unknown ordered score as $u_{k}=\left(r_{k}^{X}+r_{k}^{Y}\right) / 2$, the model $(2.1)$ is equivalent to the ridit score type quasi-symmetry (RQS) model proposed by Iki et al. (2009).

The model (2.1) can be expressed as

$$
p_{i j}=\delta^{u_{j}-u_{i}} p_{j i} \quad(i<j) .
$$

Under the model (2.1), the following properties hold:

(1) if $\delta>1$ then $F_{i}^{X}>F_{i}^{Y}$ for all $i=1, \ldots, R-1$ because $p_{i j}>p_{j i}$ for all $i<j$;

(2) if $\delta<1$ then $F_{i}^{X}<F_{i}^{Y}$ for all $i=1, \ldots, R-1$ because $p_{i j}<p_{j i}$ for all $i<j$;

(3) if $\delta=1$ then the $\mathrm{S}$ model holds because $p_{i j}=p_{j i}$ for all $i<j$.

From the above properties (1) and (2), the parameter $\delta$ of the model (2.1) can be useful for inferring whether $X$ is stochastically greater than $Y$ or vice versa.

From the above properties (1) to (3), model (2.1) constantly holds when the $\mathrm{S}$ model holds, but the converse is not necessarily true. Yamamoto et al. (2007) examined what is necessary for a model, in addition to the OQS model, to satisfy the $\mathrm{S}$ model, and gave the separation of the $\mathrm{S}$ model using the OQS and marginal mean equality (ME) models, that is, $E(X)=E(Y)$. Iki et al. (2009) also gave the separation of the S model using the RQS and ME models.

\section{Proposed models based on ordered scores and separations of symmetry}

Consider a model defined as

$$
\log \left(\frac{p_{i j}}{p_{j i}}\right)=\left\{u_{R}-\left(u_{j}-u_{i}\right)\right\} \log \theta \quad(i<j) .
$$

This model has a structure in which the $\log$ odds $\log \left(p_{i j} / p_{j i}\right)$ are inversely proportional to $u_{j}-u_{i}$. When $u_{k}$ is an equally spaced score, the model (3.1) 
is equivalent to the Another LDPS (ALDPS) model proposed by Tomizawa (1990).

We propose two original models as the ALDPS model. When $u_{k}$ can be assigned a known ordered score, such as the midpoint of the interval, we propose the Another OQS (AOQS) model as the family of model (3.1). When $u_{k}$ is assigned an unknown ordered score, such as $u_{k}=\left(r_{k}^{X}+r_{k}^{Y}\right) / 2$, we also propose the Another RQS (ARQS) model as the family of model (3.1).

The model (3.1) can be expressed as

$$
p_{i j}=\theta^{u_{R}-\left(u_{j}-u_{i}\right)} p_{j i} \quad(i<j) .
$$

It must be noted that the $u_{R}-\left(u_{j}-u_{i}\right)$, for $i<j$, is constantly positive. Under the model (3.1), the following properties hold:

(1) if $\theta>1$ then $F_{i}^{X}>F_{i}^{Y}$ for all $i=1, \ldots, R-1$ because $p_{i j}>p_{j i}$ for all $i<j$;

(2) if $\theta<1$ then $F_{i}^{X}<F_{i}^{Y}$ for all $i=1, \ldots, R-1$ because $p_{i j}<p_{j i}$ for all $i<j$;

(3) if $\theta=1$ then the $\mathrm{S}$ model holds because $p_{i j}=p_{j i}$ for all $i<j$.

From the above properties (1) and (2), the parameter $\theta$ of the model (3.1) can be useful for inferring whether $X$ is stochastically greater than $Y$ or vice versa.

From the above properties (1) to (3), the AOQS (ARQS) model constantly holds when the $\mathrm{S}$ model holds, but the converse is not necessarily true. We provide the following theorems with respect to the separations of the $\mathrm{S}$ model using the AOQS (ARQS) model. Let $U(M)$ imply that the model $M$ holds.

Theorem 1. The following necessary and sufficient condition holds:

$$
U(S) \Leftrightarrow U(A O Q S) \wedge U(M E)
$$

Proof. It is clear that the necessary condition $U(S) \Rightarrow U(A O Q S) \wedge U(M E)$ holds. We show that the sufficient condition $U(S) \Leftarrow U(A O Q S) \wedge U(M E)$ holds.

Since the ME model holds, the following equality holds:

$$
E(X)=E(Y),
$$


where $E(X)=\sum_{i=1}^{R} i p_{i}$. and $E(Y)=\sum_{i=1}^{R} i p_{. i}$. The $E(X)$ and $E(Y)$ can also be expressed as follows:

$$
\begin{aligned}
& E(X)=\sum_{s=1}^{R} \sum_{t=s}^{R} p_{t}=\sum_{s=1}^{R}\left(1-F_{s-1}^{X}\right)=R-\sum_{s=1}^{R-1} F_{s}^{X}, \\
& E(Y)=\sum_{s=1}^{R} \sum_{t=s}^{R} p_{\cdot t}=\sum_{s=1}^{R}\left(1-F_{s-1}^{Y}\right)=R-\sum_{s=1}^{R-1} F_{s}^{Y},
\end{aligned}
$$

where $F_{0}^{X}=F_{0}^{Y}=0$. Thus, the ME model can also be expressed as follows:

$$
\sum_{s=1}^{R-1} F_{s}^{X}=\sum_{s=1}^{R-1} F_{s}^{Y} .
$$

Under the AOQS model,

(1) if $\theta>1$ then $\sum_{s=1}^{R-1} F_{s}^{X}>\sum_{s=1}^{R-1} F_{s}^{Y}$ because $F_{s}^{X}>F_{s}^{Y}$ for all $s=$ $1, \ldots, R-1$

(2) if $\theta<1$ then $\sum_{s=1}^{R-1} F_{s}^{X}<\sum_{s=1}^{R-1} F_{s}^{Y}$ because $F_{s}^{X}<F_{s}^{Y}$ for all $s=$ $1, \ldots, R-1$

(3) if $\theta=1$ then $\sum_{s=1}^{R-1} F_{s}^{X}=\sum_{s=1}^{R-1} F_{s}^{Y}$ because $F_{s}^{X}=F_{s}^{Y}$ for all $s=$ $1, \ldots, R-1$.

Since the ME model holds, we obtain $\theta=1$. The proof is completed.

Theorem 2. The following necessary and sufficient condition holds:

$$
U(S) \Leftrightarrow U(A R Q S) \wedge U(M E)
$$

Theorem 3.2 can be obtained in a similar way as the proof of Theorem 3.1 .

\section{Goodness-of-fit test for proposed model}

Let $n_{i j}$ denote the observed frequency in the $(i, j)$ th cell of the table $(i, j=$ $1, \ldots, R)$. Assume that a multinomial distribution applies to the $R \times R$ table. The maximum likelihood estimates of the expected frequencies under the model can be obtained using the Newton-Raphson method in the loglikelihood equation. 
To obtain the maximum likelihood estimates of the expected frequencies under the model (3.1), we must maximize the Lagrangian

$$
\begin{aligned}
L= & \sum_{i=1}^{R} \sum_{j=1}^{R} n_{i j} \log p_{i j}-\phi\left(\sum_{i=1}^{R} \sum_{j=1}^{R} p_{i j}-1\right) \\
& -\sum_{i=1}^{R-1} \sum_{j=i+1}^{R} \psi_{i j}\left(p_{i j}-\theta^{u_{R}-\left(u_{j}-u_{i}\right)} p_{j i}\right),
\end{aligned}
$$

with respect to $\left\{p_{i j}\right\}, \phi,\left\{\psi_{i j}\right\}$, and $\theta$. It must be noted that the ordered scores $u_{k}=\left(r_{k}^{X}+r_{k}^{Y}\right) / 2(k=1, \ldots, R)$ of the ARQS model are functions of $\left\{p_{i j}\right\}$.

Each model can be tested for goodness-of-fit by means of, for example, the likelihood ratio chi-square statistic (denoted by $G^{2}$ ) with the corresponding degrees of freedom. The test statistic $G^{2}$ of model $M$ is given by

$$
G^{2}(M)=2 \sum_{i=1}^{R} \sum_{j=1}^{R} n_{i j} \log \left(\frac{n_{i j}}{\hat{m}_{i j}}\right),
$$

where $\hat{m}_{i j}$ is the maximum likelihood estimate of the expected frequency $m_{i j}$ under the model $M$. The number of degrees of freedom for the AOQS and ARQS models is $(R+1)(R-2) / 2$, which is equal to that for the LDPS, ALDPS, OQS, and RQS models. The numbers of degrees of freedom for the $\mathrm{S}$ and ME models are $R(R-1) / 2$ and 1 .

\section{Application to real data}

\subsection{Application to decayed teeth data}

Consider the data of Table 1. This dataset examined the number of decayed teeth of 349 male 18-39-year-old patients of a dental clinic in Sapporo City, from 2001 to 2005. The numbers of decayed teeth on the right and left sides were categorized as " 0 to 4 ", "5 to 8", and "9 and above". The ordered scores of the OQS and AOQS models are assigned as the midpoint scores (0-4: 2; 5-8: 6.5; 9 and above: 12), assuming that the maximum numbers of teeth on the left and right sides are 15. It may not be appropriate to assume that the difference between categories (2) and (1) is equal to the difference between categories (3) and (2), because $u_{2}-u_{1}=4.5$ and $u_{3}-u_{2}=6$. Thus, we 
consider that the OQS and AOQS models are more appropriate than the LDPS and ALDPS models for these decayed teeth data. We will apply the OQS and AOQS models to Table 1.

Table 4 gives the values of $G^{2}$ for each model. As shown in Table 4 , the $\mathrm{S}$ model fits poorly, the OQS and AOQS models fit well, and the AOQS model fits better than the OQS model. Under the AOQS model, the maximum likelihood estimate of $\theta$ is $\hat{\theta}=1.09$. Thus, the probability for the $(i, j)$ th cell, $i<j$, is estimated to be $1.09^{u_{R}-\left(u_{j}-u_{i}\right)}$ times higher than the probability for the $(j, i)$ th cell. Since $\hat{\theta}>1$, we infer that, in the case of men, the number of decayed teeth on the right side tends to be greater than on the left side.

Table 4. Values of the likelihood ratio chi-square statistic $G^{2}$ for each model applied to the data of Table 1 .

\begin{tabular}{ccc}
\hline Applied models & Degree of freedom & $G^{2}$ \\
\hline S & 3 & $8.80^{*}$ \\
OQS & 2 & 1.36 \\
AOQS & 2 & 0.35 \\
\hline * means significant at the 0.05 level.
\end{tabular}

Next, using Theorem 3.1, we consider the reason the $\mathrm{S}$ model does not hold. The value of $G^{2}(M E)$ is 7.21. As per this finding, the $\mathrm{S}$ model does not hold because the ME model does not hold rather than the AOQS model.

\subsection{Application to unaided vision data}

Consider the data of Table 2. This dataset examined 4746 students of the Tokyo University of Science for unaided distance vision, in April 1982; the dataset comprised about 10\% women. The unaided distance vision of the right and left eyes is categorized as "Best", "Second", "Third", and "Worst". We want to treat the ordered scores as unknown because of the unclear cutpoints of vision grades. Thus, we consider that the RQS and ARQS models are more appropriate than the LDPS (or OQS) and ALDPS (or AOQS) models for these unaided vision data. We will apply the RQS and ARQS models to Table 2 .

Table 5 gives the values of $G^{2}$ for each model. As shown in Table 5 , the $\mathrm{S}$ model fits poorly, the RQS and ARQS models fit well, and the ARQS model fits better than the RQS model. Under the ARQS model, the maximum likelihood estimates of the ordered scores are as follows:

$$
\hat{u}_{1}=0.16, \quad \hat{u}_{2}=0.37, \quad \hat{u}_{3}=0.53 \quad \text { and } \quad \hat{u}_{4}=0.82 \text {. }
$$


Since $\hat{u}_{2}-\hat{u}_{1}=0.21, \hat{u}_{3}-\hat{u}_{2}=0.16$ and $\hat{u}_{4}-\hat{u}_{3}=0.29$, the $\hat{u}_{k+1}-\hat{u}_{k}$ for $k=1,2,3$ are unlikely to be constant. Under the ARQS model, $\hat{\theta}=$ 0.70 . Thus, the probability for the $(i, j)$ th cell, $i<j$, is estimated to be $0.70^{u_{R}-\left(u_{j}-u_{i}\right)}$ times higher than the probability for the $(j, i)$ th cell. Since $\hat{\theta}<1$, we infer that the left eye grade of students tends to be higher than their right eye grade.

Table 5. Values of the likelihood ratio chi-square statistic $G^{2}$ for each model applied to the data of Table 2 .

\begin{tabular}{ccc}
\hline Applied models & Degree of freedom & $G^{2}$ \\
\hline S & 6 & $16.95^{*}$ \\
RQS & 5 & 6.00 \\
ARQS & 5 & 5.71 \\
\hline * means significant at the 0.05 level.
\end{tabular}

Next, using Theorem 3.2, we consider the reason the $\mathrm{S}$ model does not hold. The value of $G^{2}(M E)$ is 9.94. This finding shows that the $\mathrm{S}$ model does not hold because the ME model does not hold rather than the ARQS model.

\section{Concluding remarks}

In the proposed AOQS and ARQS models, the log odds for an observation falling in the $(i, j)$ th cell instead of the $(j, i)$ th cell, $i<j$, are inversely proportional to the difference in the ordinal scores corresponding to categories $i$ and $j$. This study also examined what is necessary for a model, in addition to the AOQS (ARQS) model, to satisfy the $\mathrm{S}$ model, and gave separations of the symmetry model using the AOQS (ARQS) and ME models. Theorems 3.1 and 3.2 explain why the $\mathrm{S}$ model does not hold.

When the differences between the midpoint scores are unlikely to be constant, the AOQS (OQS) model, rather than the ALDPS (LDPS) model, may be appropriate. In fact, for the decayed teeth data in Table 1, the differences between midpoint scores are unlikely to be constant. In regard to the unaided vision data, we should treat the ordered scores as unknown because of the unclear cut-points of vision grades. When the differences between the ordinal categories are unlikely to be constant and the cutpoints of categories are unclear, the ARQS (RQS) model, rather than the ALDPS (LDPS) model, may be appropriate. In fact, for the unaided vision data in Table 2, the differences between the estimated ordinal scores are unlikely to be constant. 
Bagheban and Zayeri (2010) considered a power parameter score as an ordered score. The power parameter score of category $k$ is defined as follows:

$$
k^{a} \quad(k=1, \ldots, R),
$$

where $a>0$. The power parameter score has the following properties:

(1) if $a<1$, then the difference of scores between $k+1$ and $k$ decreases with an increase in $k$;

(2) if $a>1$, then the difference of scores between $k+1$ and $k$ increases with an increase in $k$;

(3) if $a=1$, then the power parameter score will be equivalent to the equally spaced score.

When the differences between the ordinal categories are unlikely to be constant or the cut-points of categories are unclear, we may consider applying to the data the AOQS (OQS) model with $u_{k}=k^{a}(k=1, \ldots, R)$, in which $a$ is treated as known. For example, a grid search may be used to select the optimal value of $a$. We apply the AOQS and OQS models with $u_{k}=k^{a}$ to the data in Table 2 . The value of $a$ having minimum $G^{2}$ is searched for by moving $a$ by an increment of 0.01, from 0.05 to 5. Figure 1 shows these results. The optimal value of $a$ with minimum $G^{2}(A O Q S)=4.91$ is 0.26 (namely, $a<1$ ), and the optimal value of $a$ having minimum $G^{2}(O Q S)=6.50$ is 1.90 (namely, $a>1$ ). Since assigning different ordered scores to the same dataset is inappropriate, it is essential to discuss a method to determine $a$ in future studies.

\section{Acknowledgements}

The author would like to thank the anonymous reviewers and the editors for their comments and suggestions to improve this paper. 


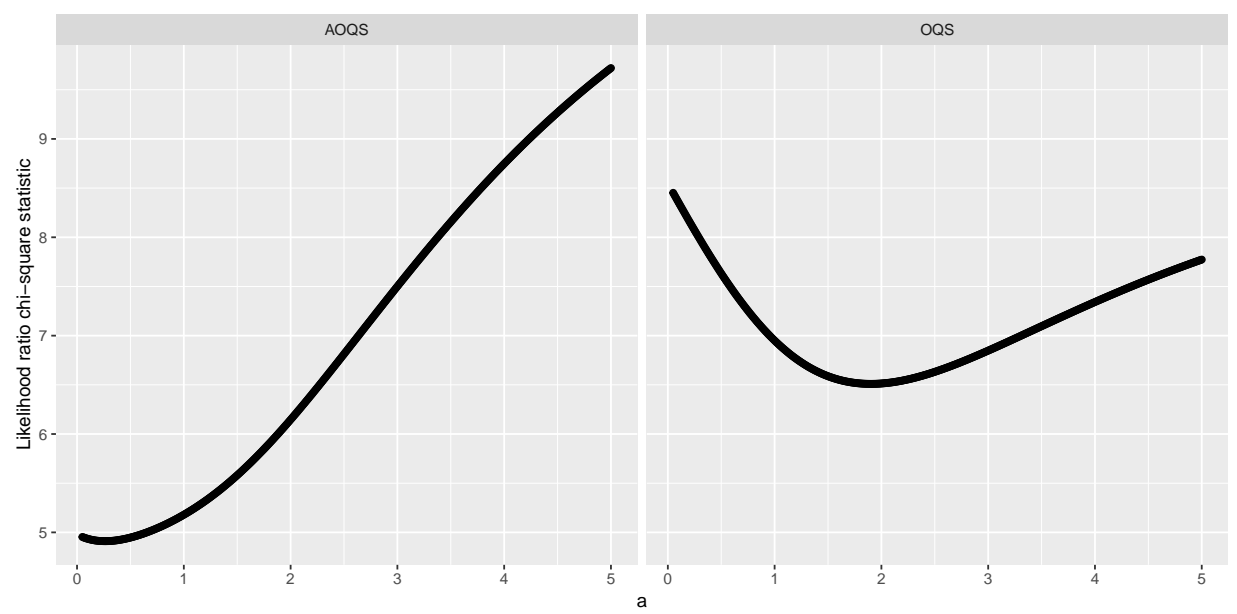

Figure 1. Values of the likelihood ratio chi-square statistic $G^{2}$ for the AOQS and OQS models with paper parameter scores $\left(u_{k}=k^{a}\right)$ applied to the data of Table 2 . The $x$-axis shows the value of $a$, and the $y$-axis shows the value of the likelihood ratio chi-square statistic of the AOQS and OQS models.

\section{REFERENCES}

Agresti A. (1983): A simple diagonals-parameter symmetry and quasi-symmetry model. Statistics and Probability Letters 1: 313-316.

Agresti A. (2002): Categorical Data Analysis, 2nd edition. Wiley, New York.

Bagheban A.A., Zayeri,F. (2010): A generalization of the uniform association model for assessing rater agreement in ordinal scales. Journal of Applied Statistics 37: 1265-1273.

Bishop Y.M.M., Fienberg S.E., Holland P.W. (1975): Discrete multivariate analysis: theory and practice. The MIT Press, Cambridge: Massachusetts.

Bowker A.H. (1948): A test for symmetry in contingency tables. Journal of the American Statistical Association 43: 572-574.

Bross I.D.J. (1958): How to use ridit analysis. Biometrics 14: 18-38.

Graubard B.I., Korn E.L. (1987): Choice of column scores for testing independence in ordered $2 \times K$ contingency tables. Biometrics 43: 471-476.

Iki K., Tahata K., Tomizawa S. (2009): Ridit score type quasi-symmetry and decomposition of symmetry for square contingency tables with ordered categories. Austrian Journal of Statistics 38: 183-192.

Senn S. (2007): Drawbacks to noninteger scoring for ordered categorical data. Biometrics 63: 296-299.

Tomizawa S. (1984): Three kinds of decompositions for the conditional symmetry model in a square contingency table. Journal of the Japan Statistical Society 14: $35-42$. 
Tomizawa S. (1990): Another linear diagonals-parameter symmetry model for square contingency tables with ordered categories. South African Statistical Journal 24: 117-125.

Tomizawa S., Miyamoto N., Iwamoto M. (2006): Linear column-parameter symmetry model for square contingency tables: Application to decayed teeth data. Biometrical Letters 43: 91-98.

Yamamoto H., Iwashita T., Tomizawa S. (2007): Decomposition of symmetry into ordinal quasi-symmetry and marginal equimoment for multi-way tables. Austrian Journal of Statistics 36: 291-306. 